Original article

\title{
Epidemiology of tuberculosis based on histopathological data and nucleic acid amplification techniques in Lebanon from January 2012 to December 2016
}

\author{
Mariam Kabbani $^{\text {a }}$, Reeda Bou Said ${ }^{\mathrm{b}, *}$, Georges Aftimos ${ }^{\mathrm{c}}$ \\ a Department of Dermatology, Lebanese University, Hadath, Lebanon \\ ${ }^{\mathrm{b}}$ Department of Ophthalmology, Lebanese University, Hadath, Lebanon \\ ${ }^{\mathrm{c}}$ Institut National de Pathologie, Baabda, Lebanon
}

\section{A R T I C L E I N F O}

\section{Keywords:}

Extrapulmonary TB

Migration

Pathology

\begin{abstract}
A B S T R A C T
Background: Tuberculosis (TB) is a worldwide disease caused by Mycobacterium tuberculosis with significant morbidity and mortality. It has variable presentations and is also influenced by host factors including immune function. Lebanon is considered to have low prevalence of this disease but has been experiencing fluctuating numbers due to several variables, migration speculated to be one of those. Our objective was to determine the epidemiology of TB in Lebanon in the interval spanning from January 2012 to December 2016. We also aimed to compare the trend of TB incidence with the trends of Syrian and foreign-worker migration.

Methods: We included 312 TB-positive patients from the National Institute of Pathology (INP) in the interval mentioned above. We studied distribution according to gender, age, regions, and the site involved.

Results: Our study showed greatest distribution of TB in Mount Lebanon (42.7\%), more cases of extrapulmonary $\mathrm{TB}$, higher incidence in females, and a mean age of 38.5 years. New TB cases increased over the years to a maximum of 69 in 2015 then decreased to 61 in 2016. This trend was similar to the trend of Syrian migration with statistically significant positive correlation.

Conclusion: There was a relationship between the Syrian migration and the increase in TB cases in Lebanon likely due to overburdening the screening protocols. We thus emphasize on the importance of the screening regimens, and enhancing and implementing them.
\end{abstract}

\section{Introduction}

Tuberculosis (TB) is an infectious disease caused by Mycobacterium tuberculosis which is transmitted through inhalation of aerosol droplets containing the bacteria. Humans are its only known hosts and it can't spread by fomites. ${ }^{1}$ It has various manifestations depending on the involved organ, lung being most common, but extrapulmonary involvement is also possible whether as a primary infection or following reactivation. $^{2}$

TB represents a major worldwide health problem with global incidence rate of 10.0 million in 2017. Two thirds of these cases originated in eight countries: India (27\%), China (9\%), Indonesia (8\%), the Philippines (6\%), Pakistan (5\%), Bangladesh (4\%), Nigeria (4\%) and South Africa (3\%). It is the primary cause of death from a single infectious agent. And although its mortality rate is decreasing, it resulted in
1.3 million deaths in 2017 not counting the 300,000 deaths in human immunodeficiency virus (HIV)-positive individuals. ${ }^{3}$

Lebanon has a low TB burden. ${ }^{4}$ In countries with low incidence of tuberculosis, most cases are foreign-borne. ${ }^{5}$ Migration is a complex phenomenon that is in constant growth where the number of migrants reached 258 million in 2017, up from 173 million in 2000. In 2016, Lebanon ranked fourth among the countries with the largest population of refugees (1.6 million). ${ }^{6}$ In addition to the huge number of refugees, Lebanon receives large numbers of foreign laborers. The total number of work permits given to foreigners almost tripled in fifteen years, from 71 , 991 to 198,452 permits; the most common of which are issued to nationals from Ethiopia and Bangladesh, both belonging to the list of the 20 high TB burden countries.

The incidence of TB reflects the developmental status of a country. The effects of the socioeconomic conditions are also evident in the

\footnotetext{
* Corresponding author. Chouit 5233, Mount Lebanon, Lebanon.

E-mail address: reedabs@gmail.com (R.B. Said).
} 
distribution of tuberculosis within the same nation. This disparity manifests in Lebanon by the presence of significant reservoirs notably in Beirut and North districts (38.4\% and $27.2 \%$, respectively). ${ }^{7}$ The rate of new reported tuberculosis cases in Lebanon increased to reach 20 per 100,000 in 2013 after a nadir of 9 per 100,000 in $2006 .^{8}$ This increase coincided with the Syrian civil war and the influx of asylum seekers into Lebanon.

In Lebanon, national TB programs are implemented to screen, diagnose and treat tuberculosis infections, but epidemiological data are scarce.

The primary objective of this study is to determine the epidemiological distribution of TB in Lebanon during the four years spanning 2012 to 2016 based on histopathological data and nucleic acid amplification techniques (NAAT), and divide them according to gender, age groups, nationality, and geographical areas. It also aims to compare the resulting data with preimmigration values.

\section{Methods}

This is an epidemiological cross-sectional study involving 312 patients from January 2012 to December 2016 in the Lebanese National Institute of Pathology (Institut National de Pathologie), which receives samples from different hospitals across Lebanon.

1. Inclusion criteria:

- Patients residing in Lebanon.

- Patients with tuberculosis proven on biopsy, or with NAAT on bronchoalveolar lavage or pleural fluid.

2. Exclusion criteria:

- Patients with nontuberculous granulomatous disease.

We used the database of the INP to find all cases of granulomatous diseases and only included definite TB cases.

Patients were classified as having tuberculosis if at least one of the following criteria was met:

- The biopsy, taken from a pulmonary or an extrapulmonary site, showed caseating granulomatous inflammation and/or positive NAAT results.

- Bronchoalveolar lavage or pleural fluid were positive for specific nucleic acid sequences by NAAT

Data concerning the numbers of Syrian migrants and foreign workers was obtained from the Ministry of Internal affairs and the Ministry of Labor respectively.

All statistical analyses were performed using IBM SPSS statistics, version 22. Categorical variables were summarized as frequency and percentage and compared using Chi-square test or fisher exact test. Continuous variables were presented as means and standard deviation. The means of quantitative variables by year were compared using Oneway ANOVA. Correlation analysis was deduced by Pearson coefficient. All tests were two-sided and p-value $<0.05$ was considered to be statistically significant.

Ethics Approval Statement: Since this is a retrospective study with databases as sources of data, ethical approval was not required.

\section{Results}

\subsection{Distribution}

\subsubsection{Yearly}

A total of 312 cases of newly diagnosed tuberculosis were diagnosed in the years spanning 2012-2016. The highest number totaling 69 (22.1\%) was recorded in 2015 followed by $66(21.1 \%)$ in 2014 . An equal value of 61 (19.6\%) was shared by the years 2013 and 2016 whereas the year 2012 had the lowest number of cases amounting to 55 (17.6\%).

\subsection{Demographics}

Among the 312 patients included, 204 were females (65.4\%) and 108 were males (34.6\%).

The mean age was 38.5 with a range of $2-89$ years.

\subsubsection{Geography}

More than third (42\%) of the total cases were located in the region of Mount Lebanon. As for cities, Saida harbored the highest percentage (9.8\%), followed by Bekaa (8.5\%) and Kesrwen (8.1\%).

\subsubsection{Tissue of origin}

Of the newly diagnosed TB cases, $85.6 \%$ were extrapulmonary and the remaining $14.4 \%$ were pulmonary. These results varied significantly ( $\mathrm{p}<0.001$, obtained using chi-square) according to the age categories. More than half $(53.4 \%)$ of the extrapulmonary tuberculosis belonged to the age group $21-40$ whereas nearly half $(47.1 \%)$ of those from pulmonary origins belonged to age group 41-60.

\subsection{Foreign population}

\subsubsection{Syrian refugees}

We collected data from the ministry of internal affairs concerning the Syrian refugees. It showed a steady increase in their cumulative number to a maximum of 1,300,000 in 2015 then decreasing to 1,050,00 in 2016 (Fig. 1). Using Pearson coefficient to test the existence of correlation with TB incidence, $r$ equaled +0.939 with a $\mathrm{p}$ value of 0.018 (Fig. 2).

\subsubsection{Foreign workers}

Data was collected from Ministry of Labor (unavailable before the year 2013). Their bulk consisted of Ethiopians, Bangladeshis, and Filipinos (Fig. 3). The number of new Ethiopian workers entering Lebanon increased dramatically in the years of our study whereas those from the Philippines witnessed a steady decrease. As for Bangladeshi workers, their numbers underwent numerous fluctuations while the rest of the foreign labor force, including Sri Lankans and Indians, dwindled markedly in numbers. Calculating the correlational relationship between Ethiopians, Bangladeshi and Filipino numbers and that of the TB incidence, we find a Pearson coefficient of $-0.285(\mathrm{p}=0.715), 0.359$ ( $\mathrm{p}$ $=0.641),-0.1(\mathrm{p}=0.9)$, respectively (Fig. 4).

\section{Discussion}

Our results show that most of the new cases of tuberculosis were clustered in the region of Mount Lebanon (42.7\%) followed by South Lebanon $(22.2 \%)$. This finding marks a change from the reservoirs located in Beirut (38.4\%) and the North districts (27.2\%) noted by Kalaajieh WK and collaborators. ${ }^{7}$ This article published in 1999 also showed higher prevalence in males (57.2\%) and in those older than 15 years of age (81\%). Our study found similar age distribution but higher

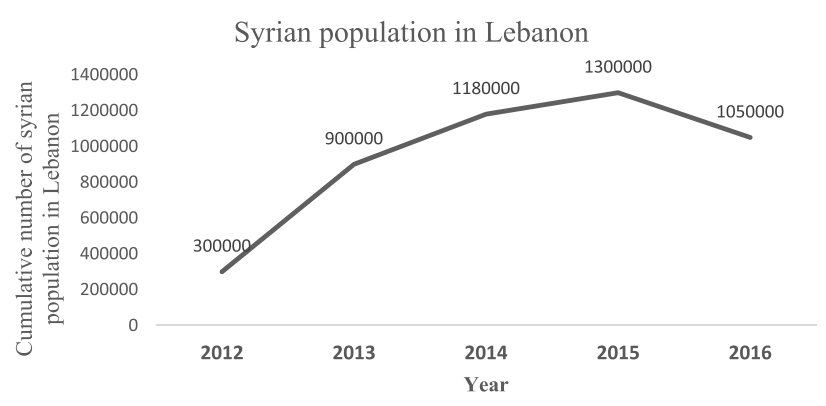

Fig. 1. Number of Syrians in Lebanon by year. Variation in the number of Syrian immigrants in the period spanning 2012 to 2016. 


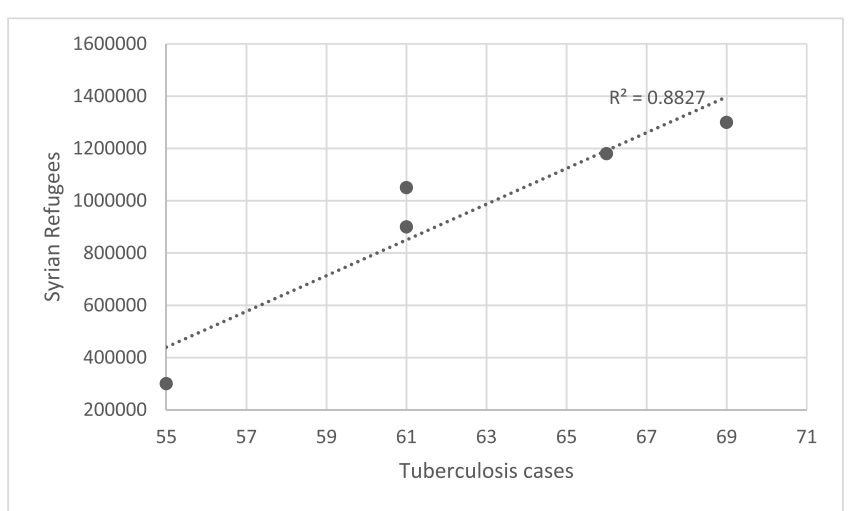

Fig. 2. Linear correlation between tuberculosis cases per year and number of Syrian refugees. Pearson coefficient $r=+0.939$, with $p=0.018$.

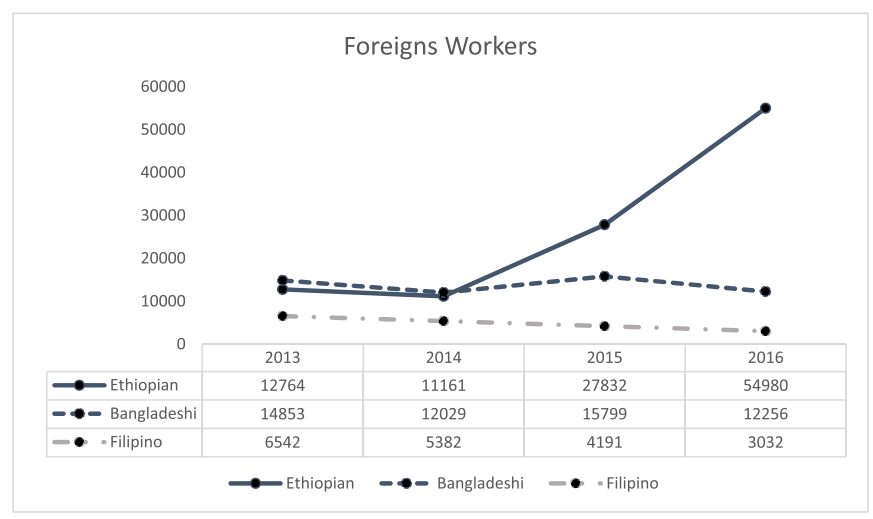

Fig. 3. Number of foreign workers in Lebanon by year. Variation of the numbers of foreign workers in Lebanon based on the numbers of the most common nationalities.

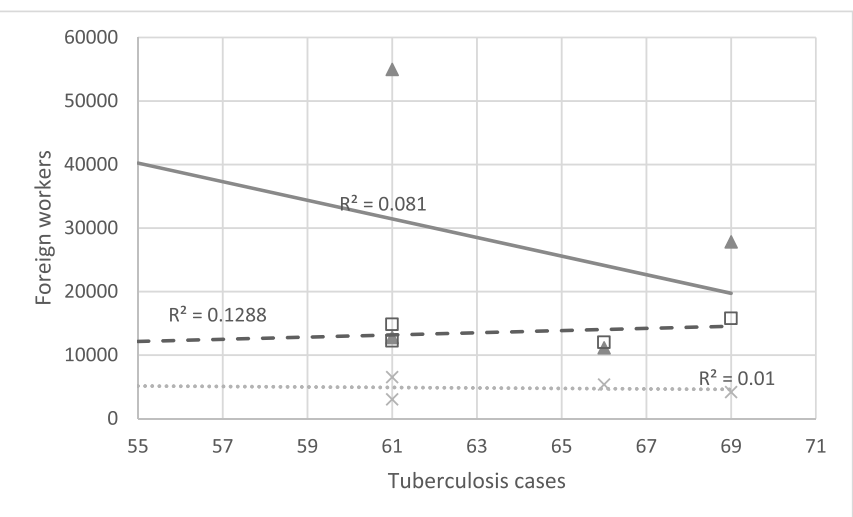

Fig. 4. Correlational relationship between tuberculosis cases per year and foreign workers of different nationalities. No statistically significant correlation can be found, with Pearson coefficient ( $r$ ) for Ethiopians of -0.285 ( $p=$ 0.715), for Bangladeshis of 0.359 ( $\mathrm{p}=0.641$ ), and for Filipinos $-0.1(\mathrm{p}=0.9)$.

prevalence in females (65.4\%).

We also noticed a higher incidence of extrapulmonary TB relative to pulmonary cases. This is probably due to the fact that our results were from a pathology center with principally tissue samples and biopsies taken from extrapulmonary sites.

The incidence of tuberculosis in Lebanon reached its lowest in 2006 with a reported value of 9 per 100,000 thus enabling Lebanon to be classified as low burden country $(<20$ per 100,000$)$. This value more than doubled in 2013 to reach a value of 20 per $100,000 .^{8}$ In our center, new cases of tuberculosis averaged 50 before the year 2010. During this study the incidence of tuberculosis followed a trend during the years 2012-2016. It increased to a maximum in 2015 and then decreased after that.

This sudden change in TB burden coincided with the Syrian crisis and influx of refugees. The decline in incidence seen after 2015 also matched the decline in numbers of Syrian refugees which could have been secondary to implementation of a new Visa law interrupting the registration of new Syrian migrants after the year 2015 and/or due to refugees returning to their country. ${ }^{9}$ Comparing this pattern with that of the fluctuation of TB cases observed in this study, a positive Pearson factor $(+0.939)$ can be inferred with a significant $p$ value of 0.018 . Thus, a correlational relationship exists between the influx of Syrian refugees and the trend followed by TB incidence in Lebanon.

A similar pattern of refugee influx coinciding with an increase in TB cases was observed in a Turkish study. In evaluating the impact of Syrian civil war on the incidence of pulmonary tuberculosis in Hatay between 2010 and 2013, Doğru and Döner noted that the highest number of all TB cases (Turkish and Syrian) was observed in 2013, the year with the highest recorded immigration in that region. The authors also described lower treatment success among Syrian refugees compared with the local Turkish population which was attributed to higher rates of loss to follow-up and lower rates of directly observed therapy in the former population. ${ }^{10}$

On the other hand, a study was conducted between 2011 and 2017 and published recently by Boudville et al., in order to identify a possible correlation between immigration and TB notification rates in Europe, an area with generally low TB incidence. This study remarked that among the 28 European Union countries, plus Norway and Iceland, 3 countries - Germany, Italy, and Norway-had significant positive correlation between the two variables. However, 8 countries had statistically significant negative correlation between $\mathrm{TB}$ rates and immigration, and the remaining 19 countries showed no significant correlation. Nevertheless, the authors stressed the importance of surveillance of TB cases and migration, and proper reporting of cases. ${ }^{11}$

Lonnroth et al. remarked that in 20 of the 30 low-incidence countries, half of the TB cases were foreign-borne in 2015. The known risk factors for TB differ along the migration course. That related to preimmigration was the ТВ burden in the country of origin. Whereas, post arrival incidence was determined by the policies in the host country regarding health and migration. Refugees and asylum seekers often face additional risk factors during migration including overcrowding, imprisonment, malnutrition, and contact with high-risk groups. ${ }^{12}$ In fact, studies have shown that among immigrants, certain groups are at higher risk for TB than others. For example, a Danish study remarked that higher-risk groups are refugees, including resettlement refugees and asylum seekers, and family-reunified to refugees. They attributed this to the circumstances of travel which favor transmission and reactivation of TB. ${ }^{13}$

The reported TB incidence by the Syrian government to WHO before the conflicts was 23 per 100,000 in 2010 . This rate remained the same in 2012 and reached 19 per 100,000 in 2017. But such stable and declining rates most likely underestimate the true tuberculosis burden because in 2016, microbiology cultures were only available in Damascus and Homs which are government-controlled cities. In addition, only 8 labs had the capacity to perform smear tests. However, in areas not under government controls, diagnostic capacities were vastly insufficient and were restricted to chest X-rays and microscopy. These limitations hinder the diagnosis of extrapulmonary and smear negative TB. ${ }^{14}$

With respect to foreign laborers, Ethiopia, Philippines, and Bangladesh are 3 of the 20 highest countries with TB burden. Incidence in 2018 reached 164 per 100,000, 554 per 100,000, and 221 per 100,000 in these three countries respectively. However, the statistical correlations with the TB incidence, be it weakly negative for both Ethiopians and Filipino workers or weakly positive for Bangladeshi 
workers, were not significant (all p values were more than 0.05 ).

These results may be secondary to the overburden of screening protocols by the sudden and large influx of Syrian refugees following the conflicts in their country which may have led to delays or incomplete implementations of these measures. Whereas concerning foreign laborers, the usual screening procedures were not subject to any new strains. This emphasizes the efficacy of the screening protocols implemented on foreign laborers and encourages more rigorous screening measures on existing and incoming migrants.

Our study has some limitations. Data was only extrapolated from one center, the INP. Although it receives samples from all over Lebanon, patterns restricted to one center may not reflect on all of Lebanon. Furthermore, after 2015, no new Syrian refugees were officially reported and thus TB was underreported in immigrants.

Due to what was mentioned above and the importance of tuberculosis studies in Lebanon, further multicentric studies are needed to evaluate the relations observed in our study.

\section{Declaration of competing interest}

The authors declare no conflict of interest and state no financial support.

\section{Acknowledgements}

We would like to thank our families for their continuous support.

We would also like to express our appreciation to the doctors at the faculty of medicine at the Lebanese University.

Our special thanks are extended to the INP residents and physicians for their guidance throughout our work on this study.

\section{References}

1 CDC. How TB Spreads | TB. New York: Centers for Disease Control and Prevention; 2016 March 11 [cited 2019 Nov 10] https://www.cdc.gov/tb/topic/basics/howtbsp reads.htm.

2 van Crevel R, Ottenhoff TH, van der Meer JW. Innate immunity to Mycobacterium tuberculosis. Clin Microbiol Rev. 2002 Apr;15(2):294-309.

3 World Health Organization. Global Tuberculosis Report 2018. Geneva: World Health Organization; 2018:231.

4 National TB programme (LB). National Guidelines for Tuberculosis Prevention, Care and Elimination in Lebanon. Beirut: Ministry of Public Health (LB); 2017:58.

5 Van Aart C, Boshuizen H, Dekkers A, Korthals Altes H. Time lag between immigration and tuberculosis rates in immigrants in The Netherlands: a time-series analysis. Int $J$ Tubercul Lung Dis. 2017 May;21(5):486-492.

6 Department of Economic and Social Affairs, Population Division (US). International Migration Report 2017: Highlights. New York: United Nations; 2017:31. Report No. ST/ESA/SER.A/404.

7 Kalaajieh WK. Epidemiology of tuberculosis in Lebanon. Int J Tubercul Lung Dis. 1999 Sep;3(9):774-777.

8 Araj GF, Saade A, Itani LY, Avedissian AZ. Tuberculosis burden in Lebanon: evaluation and current status. J Med Liban. 2016;64(1):1-7.

9 De Bel-Air F. Migration Profile: Lebanon. Issue 2017/12. Florence (ITA): European University Institute; 2017 May. 20. Report No. QM-AX-17-012-EN-N.

10 Doğru S, Döner P. Frequency and outcomes of new patients with pulmonary tuberculosis in Hatay province after Syrian civil war. Indian J Tubercul. 2017 Apr;64: 83-88.

11 Boudville DA, Joshi R, Rijkers GT. Migration and tuberculosis in Europe. J Clin Tuberc Other Mycobact Dis. 2020 Jan 7;18:100143.

12 Lönnroth K, Mor Z, Erkens C, et al. Tuberculosis in migrants in low-incidence countries: epidemiology and intervention entry points. Int J Tubercul Lung Dis. 2017 June;21(6):624-636.

13 Kristensen KL, Lillebaek T, Petersen JH, et al. Tuberculosis incidence among migrants according to migrant status: a cohort study, Denmark, 1993 to 2015. Euro Surveill. 2019;24(44), pii=1900238.

14 Abbara A, Almalla M, AlMasri I, et al. The challenges of tuberculosis control in protracted conflict: the case of Syria. Int J Infect Dis. 2019 Oct 19;90:53-59. 